\title{
Distinguishability Analysis for Multiple Mass Models of Servo Systems with Commissioning Sensors
}

\author{
Mathias Tantau ${ }^{1}$, Lars Perner ${ }^{2}$ and Mark Wielitzka ${ }^{1}$
}

\begin{abstract}
Physically motivated models of electromechanical motion systems enable model-based control theory and facilitate system interpretation. Unfortunately, the effort of modelling restricts the usage of model-based methods in many applications. Some approaches to automatically generate models from measurements choose the best model based on minimizing the residual. These model selection attempts are limited due to ambiguities in reconstructing the internal structure from the input-output behaviour because usually motion systems have only one actuator and one sensor. Often, it is unknown if the resulting model is unique or if other models with different structure would fit equally well. The set of candidate models should be designed to contain only distinguishable models but ambiguities are often unknown to the experimenter. In this paper distinguishability is investigated systematically for a class of multiple mass models representing servo positioning systems. In the analysis a new criterion for indistinguishability is used. The benefit of additional, structural sensors on distinguishability of models is demonstrated which suggests to mount them temporarily for the commissioning phase in order to facilitate the model selection. It turns out that the best results can be achieved if synergies among sensor signals are utilized.
\end{abstract}

\section{INTRODUCTION}

Several applications in the context of servo control systems require bright-grey box models with physically motivated inner structure, for example control design, feed-forward, Kalman filtering and model-based fault diagnosis.

Because modelling is time-consuming and requires considerable expert knowledge and expertise, model-based methods are not omnipresent in industry, especially in products of automation facilities, e.g. stacker cranes and positioning systems, because these products are built only in small quantities. Therefore, automatic model selection by identifying several models and choosing the one with the best fit, possibly in combination with other criteria, would be highly desirable. Such methods are called model selection or structure and parameter identification and have been described in several publications, e.g. [1], [2], [3].

Unfortunately, model selection for bright-grey box models by means of minimizing the residual is limited due to indistinguishability of structures. Different models show the exactly same input-output behaviour if the parameters are chosen suitably. No experiments exist that would lead to different residuals [4]. Ignoring this problem would lead to false interpretation, inappropriate feed-forward, erroneous diagnostics about elastic components, etc.

\footnotetext{
${ }^{1}$ Institute of Mechatronic Systems, Leibniz University Hannover, An der Universität 1, 30823 Garbsen, Germany mathias.tantaulimes.uni-hannover.de

${ }^{2}$ Lenze Automation GmbH, Am Alten Bahnhof 11, 38122 Braunschweig, Germany lars.pernerelenze.com
}

Indistinguishability can mostly be recognized from similar residuals for a given excitation [1], [5] after the identification has been carried out. A different approach is the a priori analysis of the symbolic expressions in order to prove (in)distinguishability deterministically. The latter has the advantage that identification runs of indistinguishable models can be avoided, false conclusions due to local minima can be prevented and a statement independent of the model parameters and excitation can be made. Therefore, only the latter is followed here.

Two models $M\left(\boldsymbol{p}_{0}\right)$ and $\hat{M}\left(\hat{\boldsymbol{p}}_{0}\right)$ with parameter values $\boldsymbol{p}_{0} \in \Omega$ and $\hat{\boldsymbol{p}}_{0} \in \Omega$ are said to be structurally indistinguishable if for almost every $\boldsymbol{p}_{0} \in \Omega$ there is at least one parameterization $\hat{\boldsymbol{p}}_{0} \in \hat{\Omega}$ that leads to the same output for all admissible inputs and vice versa [6]. For notational simplicity systems, which are not structurally indistinguishable, are called distinguishable in this paper. More nuanced definitions of indistinguishability and equivalence exist and can be found in e.g. [6], [7], [8], [4], [9], [10], [11].

For the analysis of linear state space models it is common to determine a minimal representation of the system called the exhaustive summary [7] or the structural invariant vector I moment invariants [6] and to investigate this set of equations for equivalent parameterizations [12], [13]. Possible minimal representations are the coefficients of the transfer function in normalized form (Laplace transform approach / transfer function approach) [14] and the Markov parameters [11]. Rather than solving for explicit expressions for the unknown parameters necessary or sufficient conditions for the existence of solutions can be tested [15], [16], [17], [18]. A different approach is to investigate the existence of a similarity transformation between the two state space models [19], but this approach is not followed here because the calculation effort has been found to be huge for the models of interest.

Existing works on this topic are mainly limited to compartmental models in biomedicine [4], [16], [13], [20], [17], [21] and often the question cannot be answered because none of the necessary or sufficient criteria is applicable. In this paper the aim is to investigate distinguishability of multiple mass models for servo systems in view of automated model selection. A new sufficient criterion is proposed. Furthermore, it is of interest if additional position/velocity/acceleration sensors can recover distinguishability when it is not given by the series sensors. These additional sensors could be mounted temporarily along the structure during the process of commissioning with little installation effort, at least in the case of acceleromenters. Finally, the existence of ambiguities 
is explained from a machine dynamics point of view.

\section{DISTINGUISHABILITY ANALYSIS}

In this section the methodology of distinguishability is explained starting with a definition of the structural invariants.

\section{A. STRUCTURAL INVARIANTS}

Starting point is the linear state space form:

$$
\dot{\boldsymbol{x}}=\boldsymbol{A}_{\mathrm{S}} \boldsymbol{x}+\boldsymbol{B}_{\mathrm{S}} \boldsymbol{u}, \quad \boldsymbol{y}=\boldsymbol{C}_{\mathrm{S}} \boldsymbol{x}+\boldsymbol{D}_{\mathrm{S}} \boldsymbol{u} .
$$

Vector $\boldsymbol{u} \in \mathbb{R}^{N_{u}}$ is the input, $\boldsymbol{x} \in \mathbb{R}^{N_{\mathrm{S}}}$ are the states and $\boldsymbol{y} \in \mathbb{R}^{N_{y}}$ are the outputs. $\boldsymbol{A}_{\mathrm{S}}, \boldsymbol{B}_{\mathrm{S}}, \boldsymbol{C}_{\mathrm{S}}$ and $\boldsymbol{D}_{\mathrm{S}}$ are analytic functions in the parameters $\boldsymbol{p} \in \mathbb{R}^{N_{\mathrm{p}}}$. It is assumed that the initial conditions are zero so that models cannot be discriminated from these and they cannot disguise differences between the models. For distinguishability analysis based on the transfer function coefficients the transfer function matrix is calculated ( $\mathbf{I}$ is the unity matrix):

$$
\boldsymbol{G}(s)=\boldsymbol{C}_{\mathrm{S}}\left(\mathbf{I} s-\boldsymbol{A}_{\mathrm{S}}\right)^{-1} \boldsymbol{B}_{\mathrm{S}}+\boldsymbol{D}_{\mathrm{S}} .
$$

The $j l$-th element has the general form:

$$
G_{j l}(s)=\frac{b_{0}+b_{1} s^{1}+\cdots+b_{n-1} s^{n-1}}{a_{0}+a_{1} s^{1}+\cdots+a_{m-1} s^{m-1}+s^{m}} .
$$

The coefficients are analytic functions of the model parameters $\boldsymbol{p}$. They depict a possible set of moment invariants $\boldsymbol{\Phi}$ if one of the coefficients is fixed, for example the denominator coefficient corresponding to the highest power of $s$ [16]. The constant coefficient can optionally be included in the set of moment invariants but usually it is not.

An alternative set of moment invariants can be obtained from the Markov parameters of the dynamic system. The transfer function is expanded as a complex power series in $s$ with infinite length [6]:

$$
\boldsymbol{G}(s)=\boldsymbol{G}_{0}+\boldsymbol{G}_{1} s^{-1}+\boldsymbol{G}_{2} s^{-2}+\ldots
$$

The set of $\left\{\boldsymbol{G}_{i}\right\}, i=0,1,2, \ldots$ can be referred to the step response $\boldsymbol{h}(t)$, resp. to the impulse response of the system $\boldsymbol{g}(t)$ and its time derivatives [22]:

$$
\boldsymbol{G}_{0}=\left.\boldsymbol{h}(t)\right|_{t=0^{+}}, \boldsymbol{G}_{i}=\left.\frac{\mathrm{d}^{i-1}}{\mathrm{~d} t^{i-1}} \boldsymbol{g}(t)\right|_{t=0^{+}}, i=1,2, \ldots
$$

Accordingly, they can be calculated from model (1):

$$
\boldsymbol{G}_{0}=\boldsymbol{D}_{\mathrm{S}}, \quad \boldsymbol{G}_{i}=\boldsymbol{C}_{\mathrm{S}} \boldsymbol{A}_{\mathrm{S}}^{i-1} \boldsymbol{B}_{\mathrm{S}}, \quad i=1,2, \ldots,
$$

leading to analytic functions in the parameters [22]. $\left\{\boldsymbol{G}_{i}\right\}, i=1,2,3 \ldots$ are called the Markov parameters.

For distinguishability analysis two models of the form (1) are considered with matrices $\boldsymbol{A}_{1}, \boldsymbol{B}_{1}, \boldsymbol{C}_{1}, \boldsymbol{D}_{1}$, resp. $\boldsymbol{A}_{2}, \boldsymbol{B}_{2}, \boldsymbol{C}_{2}, \boldsymbol{D}_{2}$ and state counts $N_{\mathrm{S} 1}$, resp. $N_{\mathrm{S} 2}$. The numbers of inputs and outputs are identical whereas the number of states are not necessarily identical. Although the series of Markov parameters is infinite, it is sufficient to consider the first $N_{\mathrm{S} 1}+N_{\mathrm{S} 2}$ Markov parameters only plus the terms $\boldsymbol{D}_{\mathrm{S} 1}, \boldsymbol{D}_{\mathrm{S} 2}$ without loss of information. This can be shown with the Cayley Hamilton theorem [11].

\section{B. Special considerations for MIMO systems}

In the case of several sensors the transition from SISO models to models with at least several outputs is of particular interest. One possibility of dealing with this is to analyse all SISO transfer functions separately and then to take the best result as the final result. This procedure will likely show the benefit of additional sensors but only in a trivial way. It is called SISO approach in the following. We are interested in situations where the result of several cooperating sensors is better than the result of the most suitable sensor evaluated in solitude. In order to investigate this the MIMO transfer function must be evaluated as a whole and the same for the Markov parameters, called MIMO approach.

When the scalar transfer function becomes a matrix, the number of coefficients increases and the computational complexity grows overproportionally for Cond. 5 and 6 in the MIMO case. It is therefore important to evaluate only as many moment invariants as necessary. For the transfer function coefficients it is known that the denominator is the same for all input-outputs pairs and consequently the denominator coefficients do not have to be evaluated several times, so the number of moment invariants increases less than proportionally with the product of inputs and outputs [18].

For the Markov parameter approach the question arises if additional system outputs allow to reduce the number of Markov parameters to evaluate. One possibility can be derived from the "generalized" Cayley Hamilton theorem, which is explained in the context of model predictive control in [23]: Starting point is the so called interaction matrix $M$ defined by the equation

$$
\begin{gathered}
\boldsymbol{A}_{\mathrm{S}}^{q}+M \boldsymbol{O}_{q}=\mathbf{0} \\
\boldsymbol{O}_{q}=\left(\begin{array}{c}
\boldsymbol{C}_{\mathrm{S}} \boldsymbol{A}_{\mathrm{S}}^{0} \\
\vdots \\
\boldsymbol{C}_{\mathrm{S}} \boldsymbol{A}_{\mathrm{S}}^{q-1}
\end{array}\right)
\end{gathered}
$$

is the observability matrix and $q \in \mathbb{N}>0$ needs to be chosen sufficiently high so that the observability matrix has full column rank and $M$ can be determined. One possible choice is $\boldsymbol{M}=-\boldsymbol{A}_{\mathrm{S}}^{q} \boldsymbol{O}_{q}^{+}$, where ${ }^{+}$denotes the pseudoinverse. Next, the $N_{\mathrm{S}} \times q N_{y}$ matrix $\boldsymbol{M}$ is partitioned into $q$ sub-matrices of size $N_{\mathrm{S}} \times N_{y}: \boldsymbol{M}=\left[\boldsymbol{M}_{1}, \boldsymbol{M}_{2}, \cdots, \boldsymbol{M}_{q}\right]$ and a matrix $\boldsymbol{\Lambda}_{i}=\boldsymbol{M}_{i} \boldsymbol{C}_{\mathrm{S}}$ is defined, $i=1,2, \ldots q$. Inserting this into (7) leads to a "generalized" Cayley Hamilton theorem

$$
\boldsymbol{A}_{\mathrm{S}}^{q}+\boldsymbol{\Lambda}_{q} \boldsymbol{A}_{\mathrm{S}}^{q-1}+\cdots+\boldsymbol{\Lambda}_{2} \boldsymbol{A}_{\mathrm{S}}+\boldsymbol{\Lambda}_{1}=\mathbf{0},
$$

in which the scalar coefficients of the standard Cayley Hamilton theorem are replaced by matrices.

For the purpose of our derivation it is useful to define matrix $\Gamma_{i}=C_{\mathrm{S}} \boldsymbol{M}_{i}$ and to multiply (7) from the left by $\boldsymbol{C}_{\mathrm{S}}$ and from the right by $\boldsymbol{B}_{\mathrm{S}}$ :

$$
\begin{gathered}
\boldsymbol{C}_{\mathrm{S}} \boldsymbol{A}_{\mathrm{S}}^{q} \boldsymbol{B}_{\mathrm{S}}+\boldsymbol{\Gamma}_{q} \boldsymbol{C}_{\mathrm{S}} \boldsymbol{A}_{\mathrm{S}}^{q-1} \boldsymbol{B}_{\mathrm{S}}+\ldots \\
+\boldsymbol{\Gamma}_{2} \boldsymbol{C}_{\mathrm{S}} \boldsymbol{A}_{\mathrm{S}} \boldsymbol{B}_{\mathrm{S}}+\boldsymbol{\Gamma}_{1} \boldsymbol{C}_{\mathrm{S}} \boldsymbol{B}_{\mathrm{S}}=\mathbf{0} .
\end{gathered}
$$


This equation will be used to limit the number of Markov parameters to evaluate for the investigation of distinguishability in the following. It is remarkable that the order $q$ can be less than $N_{\mathrm{S}}$, as for the standard Cayley Hamilton theorem, if the system has appropriate observability properties, see above.

For the derivation of distinguishability conditions an augmented system is defined as the output difference of the two systems to compare [11]:

$$
\dot{\boldsymbol{x}}_{\mathrm{a}}=\boldsymbol{A}_{\mathrm{a}} \boldsymbol{x}_{\mathrm{a}}+\boldsymbol{B}_{\mathrm{a}} \boldsymbol{u}, \quad \boldsymbol{y}=\boldsymbol{C}_{\mathrm{a}} \boldsymbol{x}_{\mathrm{a}}+\boldsymbol{D}_{\mathrm{a}} \boldsymbol{u}
$$

with

$$
\begin{gathered}
\boldsymbol{x}_{\mathrm{a}}=\left[\begin{array}{l}
\boldsymbol{x}_{1} \\
\boldsymbol{x}_{2}
\end{array}\right], \boldsymbol{A}_{\mathrm{a}}=\left[\begin{array}{cc}
\boldsymbol{A}_{\mathrm{S} 1} & \mathbf{0} \\
\mathbf{0} & \boldsymbol{A}_{\mathrm{S} 2}
\end{array}\right], \boldsymbol{B}_{\mathrm{a}}=\left[\begin{array}{c}
\boldsymbol{B}_{\mathrm{S} 1} \\
\boldsymbol{B}_{\mathrm{S} 2}
\end{array}\right], \\
\boldsymbol{C}_{\mathrm{a}}=\left[\begin{array}{ll}
\boldsymbol{C}_{\mathrm{S} 1}-\boldsymbol{C}_{\mathrm{S} 2}
\end{array}\right], \quad \boldsymbol{D}_{\mathrm{a}}=\boldsymbol{D}_{\mathrm{S} 1}-\boldsymbol{D}_{\mathrm{S} 2} .
\end{gathered}
$$

The quest for indistinguishable models has turned into a search for zero Markov parameters of the augmented system. From (10) it follows for the augmented system that all higher-order Markov parameters are zero if the first $q-1$ Markov parameters are zero because the former can be written as linear combinations of the latter. I.e. for checking distinguishability it is sufficient to check if the first $q-1$ Markov parameters and the feed-through part of the augmented system are zero. Regarding the size of $q$ : From counting the number of rows and columns in $\boldsymbol{O}_{q}$ the quantity $q$ can be low, as long as $q N_{y} \geq N_{\mathrm{S} 1}+N_{\mathrm{S} 2}$ is satisfied, but it depends on the observability properties of the augmented system. In conclusion, the number of Markov parameters can be reduced for an observable system with several outputs compared to a system with only one output.

The problem is that the augmented system is often not observable. For example, if the two systems are in fact indistinguishable, they will most likely have identical poles. Checking observability is difficult because the augmented system contains variables from both systems and a procedure similar to [19] would be required. Therefore, only the standard Cayley Hamilton theorem can be used instead of (9) which leads to the rule $q \geq N_{\mathrm{S} 1}+N_{\mathrm{S} 2}$. Then, the number of structural invariants increases proportionally with the product of inputs and outputs. Often, only the first few Markov parameters, less than required by this rule, can be calculated due to computational complexity. The implications of this limitation will become clear in the following.

\section{Necessary and sufficient criteria for structural indistin- guishability}

The following criteria for indistinguishability based on the moment invariants help to determine if a solution exists without attempting to explicitly solve for the unknowns. Unless otherwise noted they can be applied to both transfer function coefficients and Markov parameters. Cond. 1 to 5 are necessary for indistinguishability, i.e. whenever one of them is violated, distinguishability is proved and the remaining conditions can be skipped. They should be evaluated in the given order because the conditions are sorted in increasing computational complexity and because dependencies exist:

- Cond. 1: The same number of moment invariants $N_{\mathrm{MI}}$ exist (only transfer function coefficients).

- Cond. 2: The same number of coefficients exist in numerator $n$ and denominator $m$ in both models (only transfer function coefficients).

- Cond. 3: Both sets of moment invariants have the same symbolic form. The symbolic form is a representation of the moment invariants with zeros for constant elements and ones for non-constant (depending on $\boldsymbol{p}$, resp. $\hat{\boldsymbol{p}}$ ) elements [24].

- Cond. 4: The symbolic rank $N_{\mathrm{R}}$ of the Jacobian matrix $\boldsymbol{J}$ of the moment invariants with respect to the parameters is the same for both models [16], [25].

- Cond. 5: For both models the same linear dependencies among moment invariants exist [16]. These are determined by creating all possible combinations of $N_{\mathrm{R}}$ rows of $\boldsymbol{J}$, leading to as many as $\left(\begin{array}{c}N_{\mathrm{MI}} \\ N_{\mathrm{R}}\end{array}\right)$ submatrices. The row indices of those submatrices with full row rank in symbolic form are noted and must be identical for the compared systems. Constant moment invariants should be excluded to reduce calculation time. An efficient implementation based on submatrices can be found in [18].

For two models that satisfy the above necessary conditions, the following sufficient conditions for structural indistinguishability can be checked.

- Cond. 6: The moment invariants are identical in symbolic form after renaming / permutation of the variables.

- Cond. 7: The two models have as many determinable parameters as non-constant moment invariants and the constant moment invariants are identical across the two models. The number of determinable parameters equals $N_{\mathrm{R}}$ [24].

Strictly speaking, these sufficient conditions do not consider the exact domain of the parameters. It is expectable that certain models result as equivalent but the corresponding solutions for the parameters are inadmissible. Examples are non-negative parameters or prior knowledge on possible parameter ranges.

Cond. 6 tests if a mapping between the variables exists so that by renaming the variables of system 1 in $\boldsymbol{\Phi}_{1}$ the exact moment invariants of system $2 \boldsymbol{\Phi}_{2}$ are obtained and vice versa. A nontrivial case where this can be utilized is when systems are identical except for mirroring. The objective is to find a square permutation matrix $\boldsymbol{P}$ so that $\boldsymbol{p}_{1}=\boldsymbol{P} \boldsymbol{p}_{2}$. It has exactly one 1 in each row and column and otherwise zeros. Trying all possible combinations would lead to $N_{\mathrm{p}}$ ! comparisons, $N_{\mathrm{p}}$ being the number of parameters in both systems. However, often it is possible to reduce the effort by utilizing that some of the moment invariants contain only a subset of the variables. This allows to fix certain entries of the permutation matrix upfront. The procedure for determining the permutation matrix is therefore divided into two steps.

First step: The first step is defined in Algorithm 1. The priliminary permutation matrix $\boldsymbol{P}^{\prime}$ is initialized with 
all unknown and a parameter ordering is defined for both systems arbitrarily, for example lexicographic. In a loop over all moment invariants an occupancy matrix $Q$ is calculated. It will contain entries from the set $(-1,0,1)$ after line 6. In line 7 the information encoded in the existence and nonexistence of variables in the current tuple of moment invariants is incorporated into $\boldsymbol{P}^{\prime}$. At the end of the loop all pairs of moment invariants have been considered, resulting mostly in a reduced number of unknowns in $\boldsymbol{P}^{\prime}$. If at this point the permutation matrix contains rows or columns with zeros only, it can be concluded that the systems cannot be converted into each other by renaming and permuting the variables but they are not necessarily distinguishable.

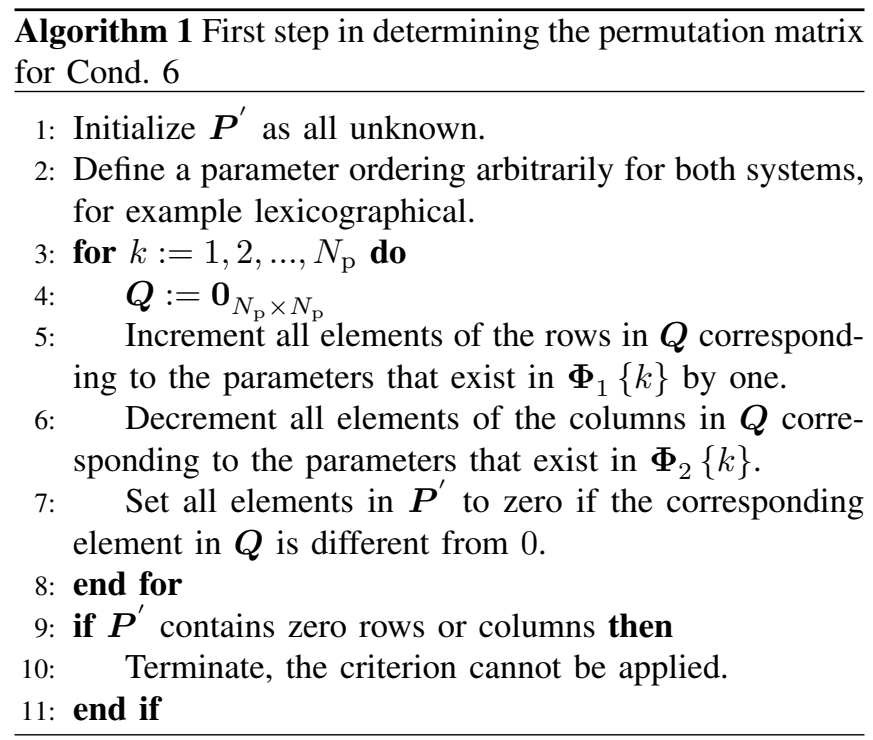

As an example from the investigations in Sec. IV the following structural invariants are given:

$$
\begin{aligned}
\boldsymbol{\Phi}_{1}= & \left(m_{1} m_{2} m_{3}\right)^{-1}\left[c_{12} c_{23}, 0, m_{3} c_{12}+m_{1} c_{23}, 0,\right. \\
& m_{1} m_{3},\left(m_{1}+m_{2}+m_{3}\right) c_{12} c_{23}, 0,\left(m_{1}+m_{2}\right) m_{3} c_{12} \\
& \left.+\left(m_{2}+m_{3}\right) m_{1} c_{23}, 0,1, c_{12} c_{23}, 0, m_{1} c_{23}\right], \\
\boldsymbol{\Phi}_{2}= & \left(m_{1} m_{2} m_{3}\right)^{-1}\left[c_{12} c_{23}, 0, m_{3} c_{12}+m_{1} c_{23}, 0, m_{1} m_{3},\right. \\
& \left(m_{1}+m_{2}+m_{3}\right) c_{12} c_{23}, 0,\left(m_{1}+m_{2}\right) m_{3} c_{12}+ \\
& \left.\left(m_{2}+m_{3}\right) m_{1} c_{23}, 0,1, c_{12} c_{23}, 0, m_{3} c_{12}\right] .
\end{aligned}
$$

They are identical except for the last element. Most of the moment invariants contain all parameters, except for number 5 and 13. For an ordering of the parameters $\left\{m_{1}, m_{2}, m_{3}, c_{12}, c_{23}\right\}$ in both systems the corresponding occupancy matrix for the thirteenth element is

$$
\boldsymbol{Q}=\left(\begin{array}{ccccc}
-1 & -1 & 0 & -1 & 0 \\
0 & 0 & 1 & 0 & 1 \\
0 & 0 & 1 & 0 & 1 \\
-1 & -1 & 0 & -1 & 0 \\
0 & 0 & 1 & 0 & 1
\end{array}\right)
$$

At the end of the first step the following preliminary permu- tation matrix results:

$$
\boldsymbol{P}^{\prime}=\left(\begin{array}{ccccc}
0 & 0 & \text { un } & 0 & \text { un } \\
0 & \text { un } & 0 & 0 & 0 \\
\text { un } & 0 & 0 & \text { un } & 0 \\
0 & 0 & \text { un } & 0 & \text { un } \\
\text { un } & 0 & 0 & \text { un } & 0
\end{array}\right) .
$$

'un' stands for unknown. Some of the rows and columns contain more than one un so that there are 4 possible permutations left.

Second step: The remaining possible combinations are iterated through and tested for equality of the moment invariants following Algorithm 2. $c_{1}$ counts the number of possible permutations, while $c_{3}$ indicates the current row in $\boldsymbol{P}$. $N_{\text {un }}$ stands for the number of unknowns in the current row and \lfloor\rfloor means rounding off to full integers. In lines 3 to 6 the current permutation is established in $\boldsymbol{P}$, which is then tested for validity in lines 7 to 9 . The termination criterion in lines 10 to 12 applies if all combinations have been tested without success. In this case no statement can be made by Cond. 6.

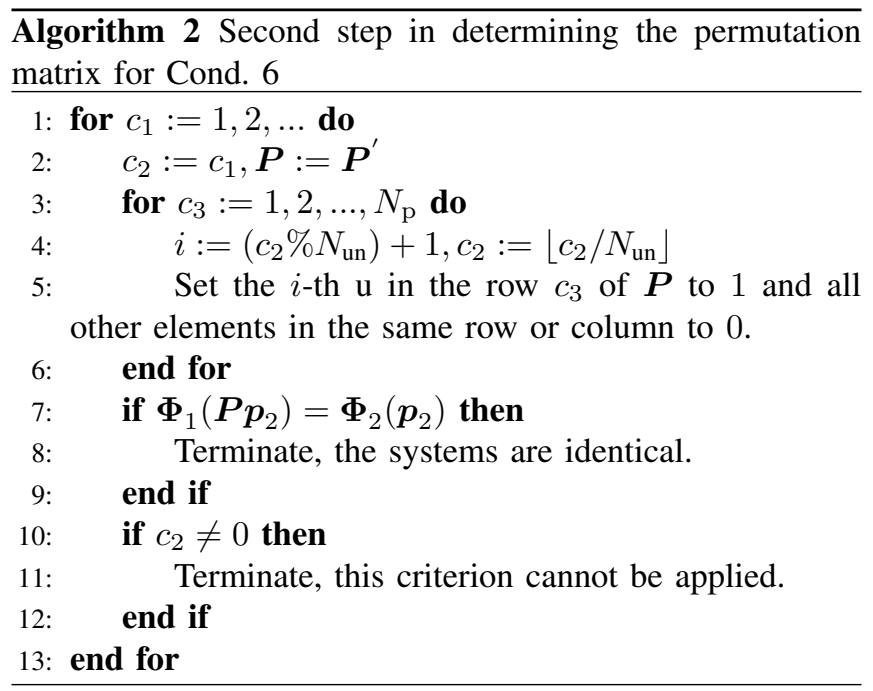

In the example the following permutation matrix results:

$$
\boldsymbol{P}=\left(\begin{array}{lllll}
0 & 0 & 1 & 0 & 0 \\
0 & 1 & 0 & 0 & 0 \\
1 & 0 & 0 & 0 & 0 \\
0 & 0 & 0 & 0 & 1 \\
0 & 0 & 0 & 1 & 0
\end{array}\right)
$$

It indicates that the two systems are identical after parameters $m_{1}$ and $m_{3}$, resp. $c_{12}$ and $c_{23}$ have been permuted.

\section{APPLICATION TO MOTION CONTROL SYSTEMS}

\section{A. Systems with one sensor}

In this section a class of multiple mass models with one sensor and one actuator is defined. It serves as a basis for the comparison with multiple sensor configurations.

For a general spring-mass-damper model with $N_{\mathrm{B}}$ masses the state space form can be obtained from the mass matrix 
$\boldsymbol{M}$, the damping matrix $\boldsymbol{D}$ and the stiffness matrix $C$ :

$$
\begin{aligned}
\boldsymbol{A}_{\mathrm{S}}^{\prime} & =\left(\begin{array}{cc}
\mathbf{0}_{N_{\mathrm{B}} \times N_{\mathrm{B}}} & \mathbf{I}_{N_{\mathrm{B}} \times N_{\mathrm{B}}} \\
-\boldsymbol{M}^{-1} \boldsymbol{C} & -\boldsymbol{M}^{-1} \boldsymbol{D}
\end{array}\right), \\
\boldsymbol{B}_{\mathrm{S}}^{\prime} & =\left(\begin{array}{c}
\mathbf{0}_{N_{\mathrm{B}} \times N_{\mathrm{B}}} \\
\boldsymbol{M}^{-1}
\end{array}\right), \\
\boldsymbol{C}_{\mathrm{S}}^{\prime} & =\left(\begin{array}{c}
\mathbf{I}_{2 N_{\mathrm{B}} \times N_{\mathrm{B}}} \\
\left(\begin{array}{cc}
\mathbf{0}_{N_{\mathrm{B}} \times N_{\mathrm{B}}} & \mathbf{I}_{N_{\mathrm{B}} \times N_{\mathrm{B}}}
\end{array}\right) \boldsymbol{A}_{\mathrm{S}}
\end{array}\right), \\
\boldsymbol{D}_{\mathrm{S}}^{\prime} & =\left(\begin{array}{cc}
\mathbf{0}_{2 N_{\mathrm{B}} \times N_{\mathrm{B}}} \\
\left(\begin{array}{cc}
\mathbf{0}_{N_{\mathrm{B}} \times N_{\mathrm{B}}} & \mathbf{I}_{N_{\mathrm{B}} \times N_{\mathrm{B}}}
\end{array}\right) \boldsymbol{B}_{\mathrm{S}}
\end{array}\right) .
\end{aligned}
$$

The actuator location as well as sensor type (position, velocity, acceleration) and location are defined by input and output multiplication matrices to select the desired entry.

For a given number of masses a variety of different models can be created depending on the position of actuator and sensor, see example in Fig. 1. These placement decisions are equivalent to the question at which positions in the structure elasticities and masses must be considered to give a suitable model. In addition, the position of the gap can be varied, which is the position between two masses without a connection by springs. This gap is known as the slideway in electric drives. In the example it is located between the first and second mass.

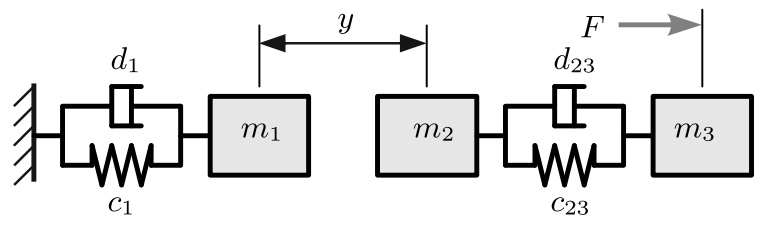

Fig. 1: Example of multipe mass model with $N_{\mathrm{B}}=3$, $n_{\text {gap }}=1, n_{\text {act }+}=3, n_{\text {act- }}=0, n_{\text {meas }+}=2, n_{\text {meas- }}=1$

In [26] the resulting combinatorics are delineated for the case of linear chains without loops or branches. Here, only the models with three masses and one sensor are shown in Tab. I. This primary sensor should be distinguished from the extra commissioning sensors that can be installed at arbitrary positions. The translational case is shown but the rotary case looks equivalent. Sec. IV focusses on these 3-mass models because more complex systems are of little practical relevance and for smaller systems the findings would mostly be trivial. Nevertheless, the methods can also be applied to other systems.

\section{B. Integration of extra sensors}

The application of additional sensors could just lead to an even more complex problem with more unknowns if it was assumed that no knowledge about the position of the sensors was available. On the other hand it is precondition that the assignment of structural elements to masses is not known a priori, so it cannot be assumed that extra sensors are located at known positions of the multiple mass models.

The following three questions depict thought experiments that are believed to require a reasonable amount of prior knowledge:
TABLE I: Complete set of 3-mass models to be considered. Collocated systems are marked by an asterisk *.

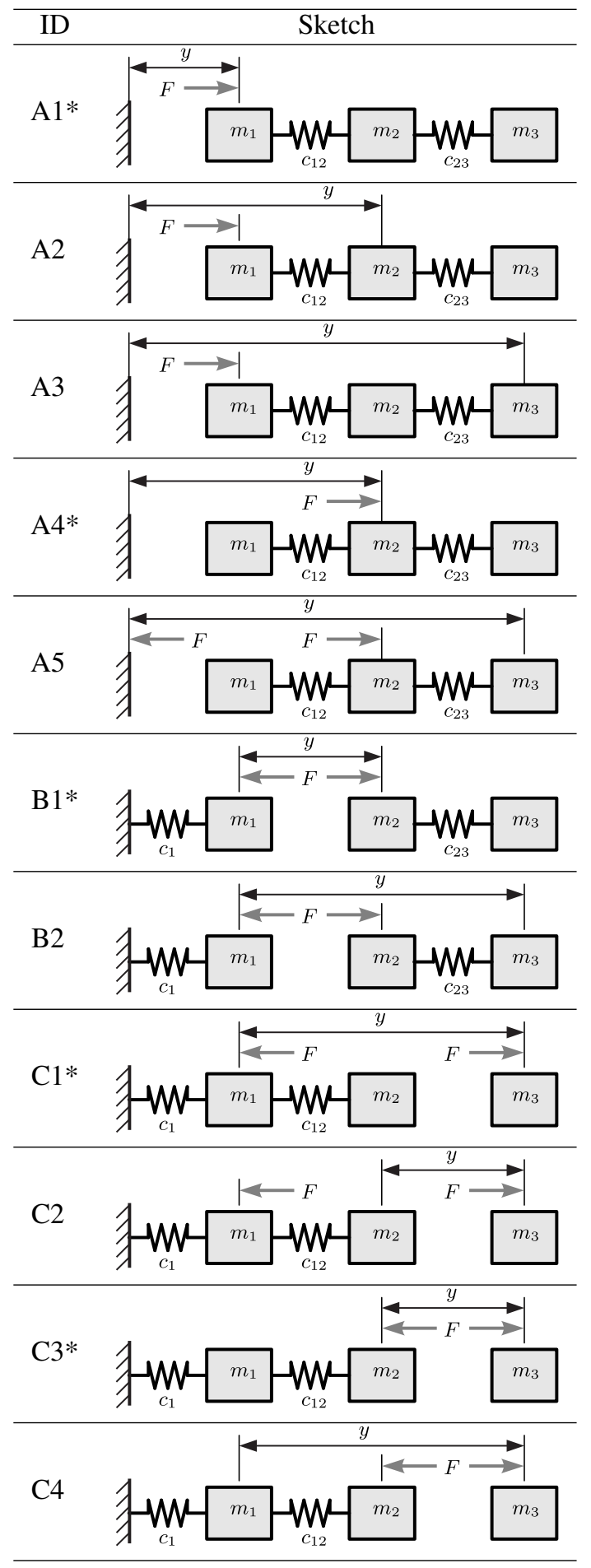

I Can indistinguishability problems be remedied by extra sensors at the left and / or right force application point?

II Can indistinguishability problems be remedied by an extra sensor at an unknown location on one of the given masses?

III With a known model, is it possible to determine the mass on which an extra sensor is located? 
Indistinguishable

Possibly indistinguishable
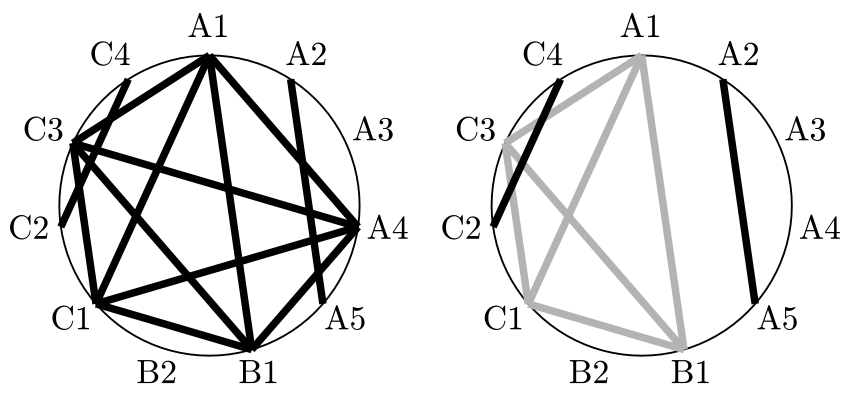

Fig. 2: Distinguishability analysis with primary sensor only. Left: damping neglected, right: damping included. Black: indistinguishable systems, grey: distinguishability question cannot be answered by the criteria from Sec. II-C

The first question is relevant because it should be possible to recognize where the main force and reaction force of the actuator are applied and to position up to two sensors at these places. The second question does not require any knowledge about the positioning of the sensor, except that the system actually behaves like a multiple mass resonator of given dimension and that the sensor is placed on one of its masses. The third question questions if the unknown sensor position can be determined from the measured time signals.

\section{RESULTS}

Distinguishability is analysed for the eleven models from Tab. I by means of the necessary and sufficient conditions from Sec. II-C applied to the transfer function coefficients and the Markov parameters. All sensors are assumed to measure accelerations. For velocity and position sensors, similar results would be obtained, except that the Markov parameters were shifted. For arbitrary combinations of different sensor types the computational effort would be different.

In Fig. 2 the distinguishability result is given with primary sensor only. Black lines represent pairs of indistinguishable systems while grey lines indicate problems where none of the above criteria could make the decision. In the inset on the left damping is neglected, while on the right a viscous damper is assumed to be connected in parallel to the displayed spring. The reason why here and in the following damping is neglected in chosen experiments is that differences caused by damping only are likely to be small and not practically helpful to distinguish systems, especially since the viscous damping model is an idealisation. For the undamped case the first 8 Markov parameters have been evaluated and in the damped case the first 7 Markov parameters. Higher numbers of Markov parameters up to $N_{\mathrm{S} 1}+N_{\mathrm{S} 2}=12$ would lead to a higher computational cost while for lower numbers more grey lines would appear.

It can be seen that many of the systems are distinguishable, but especially in the case without damping the collocated systems are not distinguishable. This is a problem because collocated systems are most relevant in practice. From a machine dynamics perspective this phenomenon can be explained by the fact that collocated 3-mass systems always show an antiresonance-resonance-antiresonance-resonance behaviour if no pole-zero cancellation occurs [27]. The exact frequencies of the resonances and antiresonances and the cross-over frequency can be adjusted by the five system parameters (3 masses and 2 spring constants). It is therefore plausible that the collocated systems without damping cannot be distinguished.

The damped systems tend to be more distinguishable. It is often not possible to come to a conclusion as Cond. 7 is not applicable in the case with damping. This is because the number of non-constant transfer function coefficients (7 . .9, 7 only for model A3) is almost always larger than the number of model parameters (7). The number of non-constant transfer function coefficients is $3 \ldots 5$ without damping and thus more likely to be equal to the number of model parameters (5). At this point the utility of the Markov parameter approach becomes evident: Distinctions A1 vs. A4, A4 vs. B1, A4 vs. C1, and A4 vs. C3 could only be made by the Markov parameters as structural invariants, Cond. 5, while the transfer function coefficients would leave unknowns here.

The reason why systems A2 and A5 are indistinguishable even when damping is considered is that A2 can be converted into A5 by mirroring about mass 2 and subsequently exchanging actors and sensors. Mirroring only permutes the parameter names but does not otherwise change the transfer function. Exchanging the sensors and actuators has no effect on the transfer function because the transfer function matrix from forces to mass positions is symmetric [27]. In this case Cond. 6 can be utilized, see example in Sec. II-C and in the damped case it is even the only criterion suitable to prove indistinguishability.

The result for question I from Sec. III-B is shown in Fig. 3 and 4 for the SISO and MIMO approach. In general, the distinguishability clearly improves leading even to the situation that all systems are distinguishable. Furthermore it can be seen that evaluating several signals simultaneously in the MIMO case allows to distinguish more systems. Without damping A2 vs. A5 cannot be distinguished in SISO because the primary sensor cannot distinguish, see above and the extra sensor at the force application point would need to distinguish A1 and A4, which is also known to be impossible. However, if both sensors are evaluated together, the simple rule of mirroring and transposition does not work for the extra sensor, so the systems become distinguishable.

The result for question II is shown in Fig. 5 for the case with damping and with 6 Markov parameters. Without damping the figure for SISO looks as in Fig. 3, left and for MIMO it is identical to Fig. 3, left, except that A1 vs. A4 is unknown. The results show that distinguishability improves even if the sensor location is not known. In this case considering the MIMO transfer functions causes the problem that more often no decision can be made due to the higher number of moment invariants to evaluate. This leads not only to longer calculation times but also makes 
Indistinguishable

Possibly indistinguishable
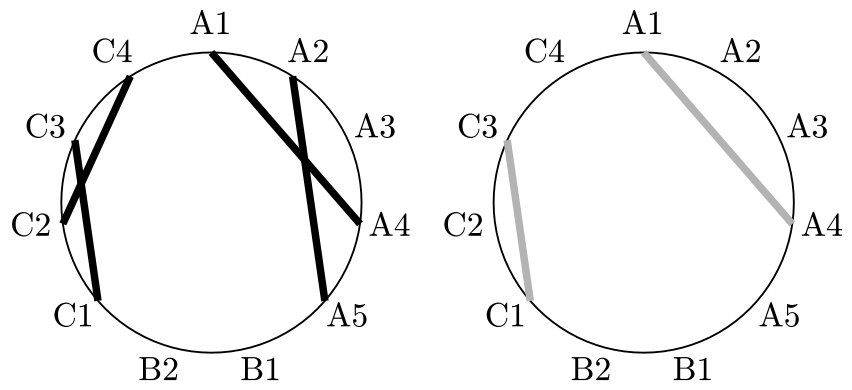

Fig. 3: Result for question I without damping, sensors on both force application points, if there are two. Left: SISO, Right: MIMO

Indistinguishable Possibly indistinguishable
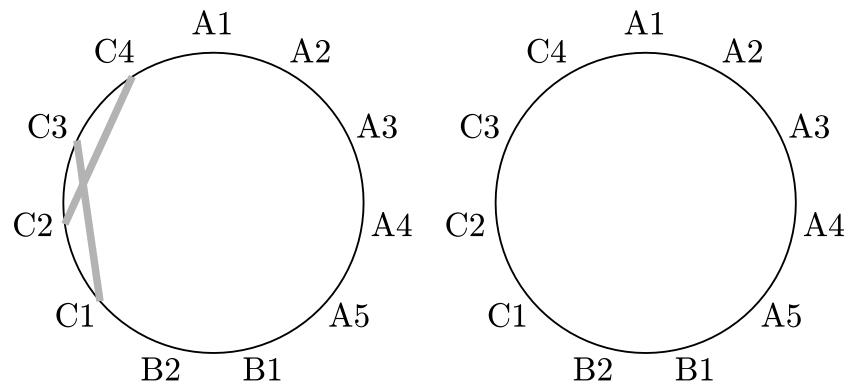

Fig. 4: Result for question I with damping. Left: SISO, Right: MIMO

Cond. 7 not applicable in many cases.

The result of question III is given in Tab. II. The result is the same with and without damping. For the eleven systems to investigate it is indicated by black crosses at which sensor position it must be expected that the correct mass cannot be determined from the sensor signal. It can be seen that for system A4 the sensor positions on masses 1 and 3 can never be distinguished and that for A5 this problem can be remedied by utilizing both sensors together. The reason is that systems A4 and A5 are symmetric, except for the primary measurement at system A5. Only in the MIMO case the signal of the sensor with unknown position can be compared with the signal of the known, primary sensor. For general parameterizations without identical poles etc. the correct mass will result from this comparison.

\section{DISCUSSION}

In this contribution distinguishability of multiple mass models representing electric drives with coupled mechanics has been analysed with focus on the utility of supplementary commissioning sensors.

The results suggest that under certain conditions, see above, (almost) all uncertainty about the model structure can be overcome and it seems that the appropriate model can be selected for any mechanical system by identifying several models and choosing the one with lowest residual. The model
Indistinguishable Possibly indistinguishable
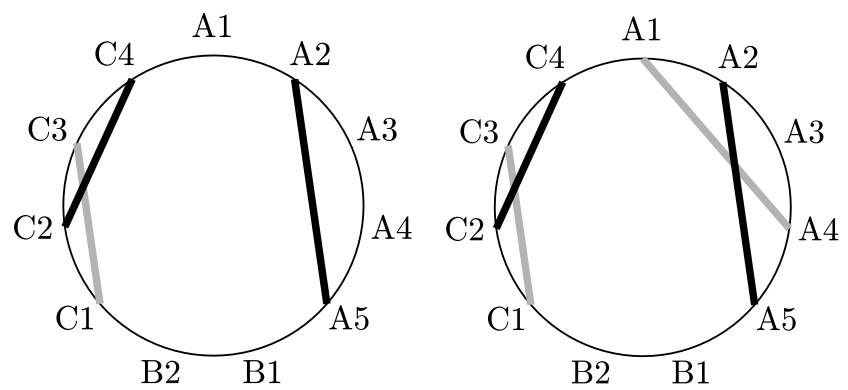

Fig. 5: Result for question II with damping. Left: SISO, Right: MIMO

TABLE II: Result for the third question with and also without damping.

\begin{tabular}{c|ccc|ccc} 
& \multicolumn{3}{|c|}{ SISO } & \multicolumn{3}{c}{ MIMO } \\
\hline mass & 1 & 2 & 3 & 1 & 2 & 3 \\
\hline A1 & & & & & & \\
\hline A2 & & & & & & \\
\hline A3 & & & & & & \\
\hline A4 & $\times$ & & $\times$ & $\times$ & & $\times$ \\
\hline A5 & $\times$ & & $\times$ & & & \\
\hline B1 & & & & & \\
\hline B2 & & & & & \\
\hline C1 & & & & & \\
\hline C2 & & & & & \\
\hline C3 & & & & & \\
\hline C4 4 & & & & & \\
\hline
\end{tabular}

selection procedure is not limited to 3-mass systems but could simultaneously determine the optimal model complexity by including information criteria, cross validation, etc. [28]. However, the most important premise should not be disregarded: The system to be investigated must resemble at least one of the candidate models. Reasons why this might not be the case are nonlinearity, such as friction, backlash, and hysteresis, unmodelled dynamics, elastic bending modes rather than discrete masses, position dependencies. For the placement of sensors on the different masses it is essential that these masses can be assigned a certain component of the system.

Furthermore, the situation becomes more complex if the variety of model structures is extended. Obvious extensions would be combined rotatory and translational systems or systems with branching, as for example storage and retrieval systems with more than one slideway. Clearly, the set of candidate models would quickly become so large that a preselection based on prior knowledge would become necessary. The automatized model selection would only depict one step in the whole procedure and should be preceded by an analysis of some candidate models with the distinguishability criteria from above.

Finally, it shall be noted that structurally distinguishable 
pairs of models can be hard to distinguish in a given experiment. Especially if the excitation is unsuitable, the difference in the measurements assigned to different underlying structures of systems can be zero or at least less than the sensor noise. In this case a chance of false identification remains, even for structurally distinguishable models.

\section{CONCLUSION}

Distinguishability of state space models was investigated for a class of multiple mass systems that represent electromechanical motion control units. Special focus was laid on the use of extra sensors, such as IMUs, that could be mounted at different positions temporarily during the commissioning to aid the model selection. The analysis was limited to a special class of 3-mass systems but could equally well be extended to models with different structure.

It was found that the extra sensors can resolve the indistinguishability problem in many cases, especially when they coincide with the force application points, but also when they are mounted at arbitrary, unknown positions along the structure. It is even possible to determine the position of a sensor from its time-series measurements in almost all cases.

Evaluating several sensors simultaneously leads to better results than combining the results obtained from several sensors evaluated in solitude. This demonstrates the effectiveness of temporary commissioning sensors due to synergies among different sources of information.

The proposed sufficient criterion for indistinguishability of models was shown to prove indistinguishability in a few cases where other criteria could not be applied.

Sometimes, systems can only be distinguished if damping is considered and the resulting differences are small. Especially in those cases practical applicability of the methods should be investigated in further works.

\section{ACKNOWLEDGMENT}

This work was sponsored by the German Forschungsvereinigungen Antriebstechnik e.V. (FVA) and the AiF Arbeitsgemeinschaft industrieller Forschungsvereinigung "Otto von Guericke“ e.V.

\section{REFERENCES}

[1] K. P. Burnham and D. R. Anderson, Model selection and inference - a practical use of the information-theoretic approach. New York: Springer, 1998.

[2] J. Aguilar, M. Cerrada, and A. T. F. Cordero, "Genetic programmingbased approach for system identification," Advances in Fuzzy Systems and Evolutionary Computation, Artificial Intelligence, pp. 329-334, 2001.

[3] M. Tantau, E. Popp, L. Perner, M. Wielitzka, and T. Ortmaier, "Model selection ensuring practical identifiability for models of electric drives with coupled mechanics," in 21st International Federation of Automatic Control (IFAC) World Congress, Berlin, Germany, 12.-17. Jul 2020.

[4] M. J. Chapman and K. R. Godfrey, "A methodology for compartmental model indistinguishability," Mathematical biosciences, vol. 96, no. 2, pp. 141-164, 1989.

[5] J. Diard, "Bayesian model comparison and distinguishability," in International Conference on Cognitive Modeling (ICCM 09), Manchester, United Kingdom, 2009, pp. 204-209.

[6] S. Vajda, "Structural equivalence of linear systems and compartmental models," Mathematical Biosciences, vol. 55, no. 1-2, pp. 39-64, 1981.
[7] E. Walter, Y. Lecourtier, and J. Happel, "On the structural output distinguishability of parametric models, and its relations with structural identifiability," IEEE Transactions on Automatic Control, vol. 29, no. 1, pp. 56-57, 1984.

[8] K. R. Godfrey and M. J. Chapman, "The problem of model indistinguishability in pharmacokinetics," Journal of pharmacokinetics and biopharmaceutics, vol. 17, no. 2, pp. 229-267, 1989.

[9] T. V. Avdeenko and S. A. Kargin, "The problem of distinguishability of state space models," in 5th International Conference on Actual Problems of Electronic Instrument Engineering Proceedings. APEIE2000. IEEE, 2000, pp. 77-82.

[10] P. Rosa and C. Silvestre, "On the distinguishability of discrete linear time-invariant dynamic systems," in 50th IEEE Conference on Decision and Control and European Control Conference. Orlando, FL, USA: IEEE, 12-15 Dec 2011, pp. 3356-3361.

[11] K. M. D. Motchon, K. M. Pekpe, J.-P. Cassar, and S. De Bièvre, "On the input-output distinguishability of single output continuous linear time-invariant systems," IEEE Transactions on Automatic Control, vol. 61, no. 7, pp. 1906-1911, 2015.

[12] A. Raksanyi, Y. Lecourtier, E. Walter, and A. Venot, "Identifiability and distinguishability testing via computer algebra," Mathematical biosciences, vol. 77, no. 1-2, pp. 245-266, 1985.

[13] K. R. Godfrey, M. J. Chapman, and S. Vajda, "Identifiability and indistinguishability of nonlinear pharmacokinetic models," Journal of pharmacokinetics and biopharmaceutics, vol. 22, no. 3, pp. 229-251, 1994.

[14] R. Bellman and K. J. Åström, "On structural identifiability," Mathematical biosciences, vol. 7, no. 3-4, pp. 329-339, 1970.

[15] K. R. Godfrey and M. J. Chapman, "Identifiability and indistinguishability of linear compartmental models," Mathematics and Computers in Simulation, vol. 32, no. 3, pp. 273-295, 1990.

[16] L.-Q. Zhang, J. C. Collins, and P. H. King, "Indistinguishability and identifiability analysis of linear compartmental models," Mathematical biosciences, vol. 103, no. 1, pp. 77-95, 1991.

[17] N. D. Evans, M. J. Chappell, M. J. Chapman, and K. R. Godfrey, "Structural indistinguishability between uncontrolled (autonomous) nonlinear analytic systems," Automatica, vol. 40, no. 11, pp. 19471953, 2004

[18] N. R. Davidson, K. R. Godfrey, F. Alquaddoomi, D. Nola, and J. J. DiStefano III, "Disting: A web application for fast algorithmic computation of alternative indistinguishable linear compartmental models," Computer Methods and Programs in Biomedicine, vol. 143, pp. 129135, 2017.

[19] T. Avdeenko and S. Kargin, "A computer algebra method for testing structural distinguishability of state space models," in 5th KoreaRussia International Symposium on Science and Technology (KORUS), vol. 1. IEEE, 2001, pp. 77-80.

[20] E. Walter and L. Pronzato, "On the identifiability and distinguishability of nonlinear parametric models," Mathematics and computers in simulation, vol. 42, no. 2-3, pp. 125-134, 1996.

[21] J. W. T. Yates, R. D. O. Jones, M. Walker, and S. Y. A. Cheung, "Structural identifiability and indistinguishability of compartmental models," Expert opinion on drug metabolism \& toxicology, vol. 5, no. 3, pp. 295-302, 2009.

[22] S. Hatakeyama, Y. Pan, and K. Furuta, "Identification of continuous systems via markov parameters and time moments," IFAC Proceedings Volumes, vol. 32, no. 2, pp. 3998-4003, 1999.

[23] M. Q. Phan, R. K. Lim, and R. W. Longman, "Unifying input-output and state-space perspectives of predictive control," Department of mechanical and aerospace engineering technical report, no. 3044, 1998.

[24] S. Vajda, "Structural equivalence and exhaustive compartmental modeling," Mathematical biosciences, vol. 69, no. 1, pp. 57-75, 1984.

[25] S. Vajda and H. Rabitz, "Identifiability and distinguishability of firstorder reaction systems," The Journal of Physical Chemistry, vol. 92, no. 3, pp. 701-707, 1988.

[26] M. Tantau, C. Helmke, L. Perner, and M. Wielitzka, "Distinguishability study of 3-mass models for electromechanical motion systems," International Journal of Modelling, Identification and Control, 2020, in press.

[27] D. J. Ewins, Modal testing: theory, practice, and application, 2nd ed. Research Studies Press Ltd., 2000.

[28] L. Ljung, System identification - theory for the user, 2nd ed. Prenticehall, 1999. 